\title{
EARLY ABNORMAL AEEG ACTIVITY IS ASSOCIATED WITH ADVERSE SHORT-TERM OUTCOME IN PREMATURE INFANTS
}

\author{
K. Mitsakis, V. Soubasi, K. Sarafidis, E. Diamanti, M. Griva, V. Drossou \\ 1st Dept of Neonatology, Aristotle University of Thessaloniki, Hippokration Hospital, Thessaloníki, Greece
}

\begin{abstract}
Aim: To identify specific early aEEG features that could be used as prognostic markers for severe brain injury in premature infants.
\end{abstract}

Methods: In 115 preterm infants, 25-32 wks' GA at birth, the aEEG recordings during the first 72 hours of life were correlated with head ultrasonographic(HUS) findings. Continuity(Co), sleep-wake cycling(Cy) and lower border(LB) of the aEEG were evaluated by semiquantitative analysis, applying pre-established criteria. The brain ultrasound was classified as seriously abnormal [intraventricular hemorrhage(IVH) 3-4, periventricular leucomalacia(PVL)], mildly abnormal (IVH 1-2, mild/moderate echodensities) or normal.

Results: The infants were divided in four groups based on brain injury severity. Group A ( $\mathrm{n}=72$, normal HUS), group B ( $n=16$, IVH 1-2 or mild/ moderate echodensities), group $\mathrm{C}(\mathrm{n}=21, \mathrm{IVH} 3-4)$ and group $\mathrm{D}$ $(n=6, P V L) .18$ infants (16 of group $C$ and 2 of group D) died during hospitalisation. Significantly lower values of all aEEG features were found in group $\mathrm{C}$ infants [Co(p:0.003), Cy(p:0.003), LB(p:0.046)]. The presence of pathological tracings (burst-suppression, continuous low voltage, isoelectric activity) or discontinuous low-voltage, the absence of $\mathrm{Cy}$ and $\mathrm{LB}<3 \mu \mathrm{V}$ in the initial aEEG displayed a sensitivity of $88.9 \%, 63 \%$ and $51.9 \%$ respectively, for severe brain injury or death. Logistic regression analysis of aEEG features after controlling for any potential confounding influence of GA, revealed significant relationship between $\mathrm{Co}$ and the presence of severe injury $(83.19 \%$ of cases with early adverse outcome could correctly classified using Co).

Conclusion: Pathological tracings or discontinuous low-voltage in the initial aEEG are predictive for poor short-term outcome in VLBW neonates. 